\title{
MEMS WING TECHNOLOGY FOR A BATTERY-POWERED ORNITHOPTER
}

\author{
T.Nick Pornsin-sirirak, S.W. Lee, H. Nassef*, J. Grasmeyer**, Y.C. Tai, C.M. Ho*, M. Keennon** \\ Caltech Micromachining Laboratory, 136-93, Pasadena, CA 91125 \\ * Mechanical and Aerospace Engineering, University of California, Los Angeles, CA 90095 \\ ** MAV Program, AeroVironment Inc., Simi Valley, CA 93063
}

\begin{abstract}
The objective of this project is to develop a batterypowered ornithopter (flapping-wing) Micro Aerial Vehicle (MAV) with MEMS wings. In this paper, we present a novel MEMS-based wing technology that we developed using titanium-alloy metal as wingframe and parylene $\mathrm{C}$ as wing membrane. MEMS technology enables systematic research in terms of repeatablility, size control, and weight minimization. We constructed a high quality low-speed wind tunnel with velocity uniformity of $0.5 \%$ and speeds from $1 \mathrm{~m} / \mathrm{s}$ to $10 \mathrm{~m} / \mathrm{s}$. We have tested and have studied the unsteady-state aerodynamics of various types of MEMS wings. Finally, we built lightweight ornithopters with electricpowered transmission system and have demonstrated successful free flights of with flight duration ranges from 5 to 18 seconds.
\end{abstract}

\section{INTRODUCTION}

We started this project with two difficult constraints. The first constraint was that the flyer must be a MAV and, by definition, MAV must have a total wingspan less than $15 \mathrm{~cm}$. The second constraint was that the flyer must fly by flapping wings (an ornithopter). Unfortunately, aerodynamics of flapping-wing flight, especially MAV size, is still not a fully-explored subject. There have been studies of insect flights [1-3]; however, unlike fixed-wing aerodynamics, there have not been any available design rules for flapping-wing aerodynamics for MAV size. As a result, we believe that there are two approaches for this project. One is to learn from natural MAV flyers and try to mimic them. The other is to study flapping-wing aerodynamics ourselves and try to improve them.

From our analysis of natural MAV flyers, we find that the MAV size falls within the range of small birds, bats, hummingbirds, and large insects [4]. Figure 1 shows some samples of natural flyers. We estimate that these flyers of MAV size weigh about 7-10 grams and we believe our ornithopter should weigh about the same. Shown in Figure 2 is statistical data on the speed vs. size relationship from a wide range of birds [5]. The general statistical tendency shows that the flight speed can be approximately given by:

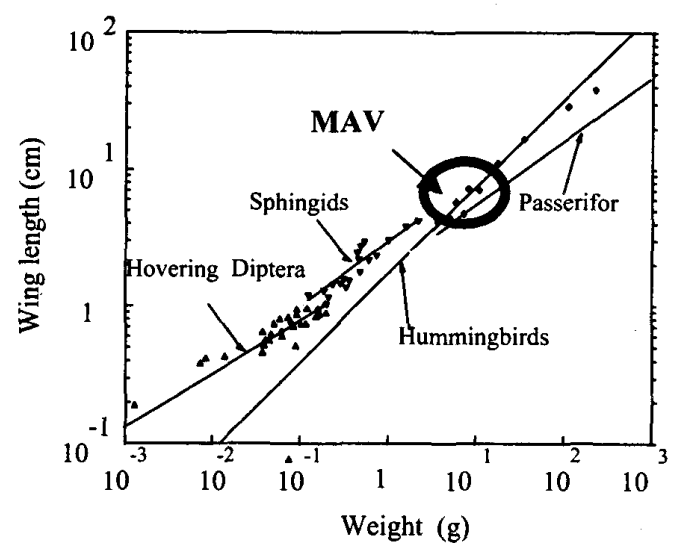

Figure 1: Size of natural flyers

$$
U=4.77 m^{\frac{1}{6}}
$$

where $U$ is the flight speed in $\mathrm{m} / \mathrm{s}$ and $\mathrm{m}$ is the mass in grams. From statistical data on wing beat rate vs. wing length [4], and wing beat rate vs. mass [6] for birds and insects, a relationship of wingtip speed, $U_{\text {vertical, and }}$ mass can be derived and are given by these following relations:

$$
\begin{aligned}
& U_{\text {vertical }} \text { upper }=11.7 m^{-0.065} \\
& U_{\text {vertical }} \text { lower }=9.6 m^{-0.043}
\end{aligned}
$$

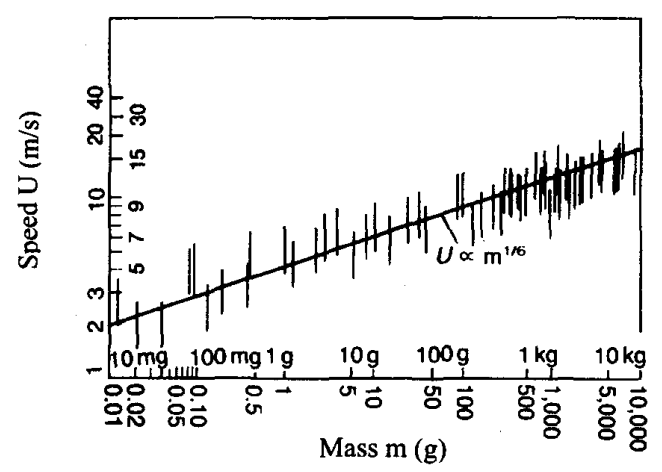

Figure 2: Flight speed of birds $\lceil 5\rceil$ 


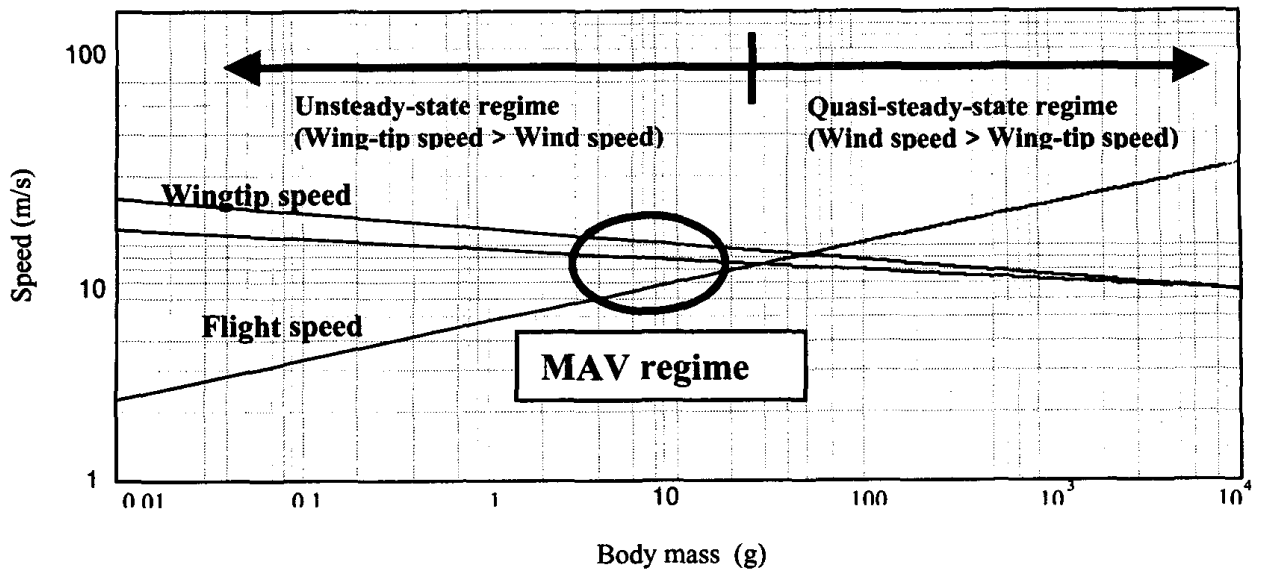

Figure 3: Flight regime of steady-state and unsteady-state of natural flyers

Combining equations (1) to (3), a plot of wingtip speed and flight speed vs. mass of insects and birds can be obtained as shown in Figure 3. The flight of flyers can be separated into two regimes: quasi-steady and unsteady states. For larger flyers, their flights can be approximated by quasi-steady-state assumptions because their wings flap at lower frequency during cruising. This means the wingtip speed is low compared to the flight speed. Thus larger birds, such as eagles and seagulls, tend to have a soaring flight. Their wings behave closely to fixed-wings. On the other hand, smaller birds and insects fly in an unsteady-state regime in which their wingtip speed is faster than their flight speed. From Figure 3, we conclude that our MAV ornithopter (mass 7-10 grams) operates in an unsteady-state flow regime. The airflow over the wings is not constant in time and cannot be approximated by quasi-steady-state assumptions. During the unsteadystate flight, the airflow is separated from the wing at the leading edge and a separation "bubble" is formed during downstroke to generate a high lift during flight [7]. The vortex bubble is formed as the stagnation streamline rolls over the leading edge. This bubble continues to grow during the downstroke and is shed at the start of the upstroke.

Thus, one of the most difficult and challenging tasks is to design and develop a highly efficient wing that has an unsteady-state aerodynamic advantage. The wing must be light and strong. In addition, it also has to be able to withstand high flapping frequency without breaking and is capable of generating enough lift and thrust to fly the prototype vehicles.

\section{DESIGN AND FABRICATION}

Since our first approach is to learn from natural flyers and mimic them, we first designed our wings based on bat wings and other insect wings, such as beetle and dragonfly wings. We developed a MEMS fabrication process using silicon and parylene $\mathrm{C}$ to make wingframe and membrane, respectively. These fabricated wings are shown in Figure 4. The bone width of the bat wing is $350 \mu \mathrm{m}$ and the membrane thickness for both wings is $15 \mu \mathrm{m}$. However, silicon wingframes were too fragile. They broke easily. Therefore, we have developed a new process using titanium-alloy metal as wingframe instead.

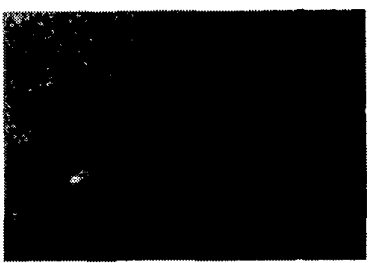

a) Silicon bat wing

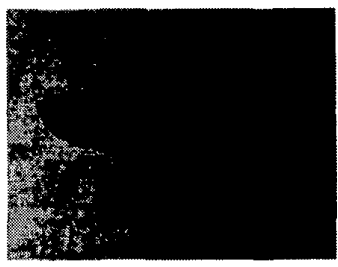

b) Silicon dragonfly wing
Figure 4: MEMS fabricated silicon wings

\section{Titanium-alloy Metal Wings}

We have experimented with various materials for wingframe structure. For example, aluminum metal is light in weight but it is too soft. Stainless steel is strong but its density is twice as high as that of titanium-alloy metal. The etchant solution $\left(\mathrm{FeCl}_{3}\right)$ is dark brown in color and is impossible to judge when the etching is finished. Besides, the etching process of stainless steel must be performed at a high temperature in order to yield a reasonable etching rate.

We have chosen titanium-alloy metal for several reasons. First, it is light and strong. Second, it can be easily tapered to vary the thickness of wingspars. Third, since titanium-alloy is ductile, it can be bent to create wing camber to improve performance. Finally, the etching process of titanium-alloy can be conducted at room temperature with reasonable etching rate. For 
wing membrane, parylene $\mathrm{C}$ is selected because it can be deposited directly onto titanium-alloy at any desired thickness. Its adhesion to titanium-alloy is excellent. Moreover, it is light, strong, and can withstand high flapping frequency of more than $30 \mathrm{~Hz}$ without tearing. Lastly, parylene $\mathrm{C}$ is deposited at room temperature and yields a conformal coating. Thus step corners are uniformly covered. Figure 5 shows various fabricated titanium-alloy MEMS wings, ranging from insect wings to simple spar wings. Table 1 shows selected mechanical properties of both titanium-alloy metal and parylene $\mathrm{C}$.

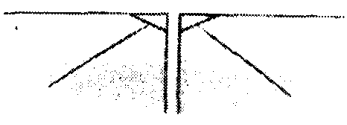

a) simple

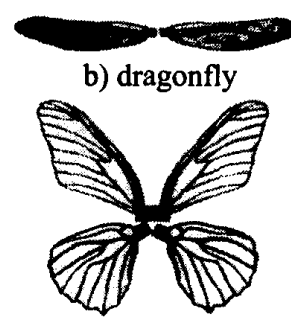

c) butterfly

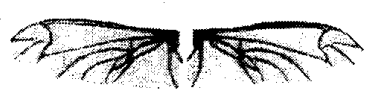

d) beetle

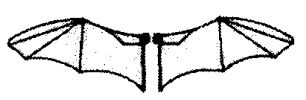

e) bat

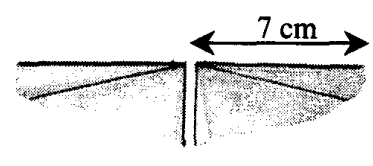

f) $\mathrm{CIT} 7 \mathrm{x} 3 \mathrm{~S} 20$
Figure 5: Titanium-alloy MEMS wings

Table 1: Mechanical Properties of Ti-6Al4V [8] and Parylene C [9]

\begin{tabular}{|l|c|c|}
\hline Properties & Ti-6Al4V & Parylene C \\
\hline Density, $\mathrm{g} / \mathrm{cm}^{3}$ & 4.5 & 1.3 \\
\hline Young's Modulus, $\mathrm{GPa}$ & 110 & 3 \\
\hline Tensile Strength, $\mathrm{MPa}$ & 100 & 70 \\
\hline Yield Strength, $\mathrm{MPa}$ & 97 & 56 \\
\hline $\begin{array}{l}\text { Coefficient of Thermal } \\
\text { Expansion, } \mathrm{x} 10^{-5} /{ }^{\circ} \mathrm{C}\end{array}$ & 0.9 & 3.5 \\
\hline
\end{tabular}

\section{Fabrication process}

We have tried to use a conventional method, i.e. using carbon fiber rod, mylar, and glue, to make wings. However, we found that making wings that way is cumbersome. Glue also adds significant weight. Moreover, identical wings are difficult to achieve unless a mold is made. This is costly, time-consuming, and slow. This method cannot accommodate effectively the study of the design variable changes.

For many reasons we claim the new MEMS wing technology is necessary because MEMS wings enable systematic research in term of repeatability, size control, weight minimization, mass production, and fast turnaround time. Moreover, complicated structures, such as dragonfly, butterfly, and beetle wings, as shown in Figure $5 \mathrm{~b}$ ), c) and d), respectively, can be easily fabricated using photolithography technology. Figure 6 shows the fabrication process of titanium-alloy MEMS wing.

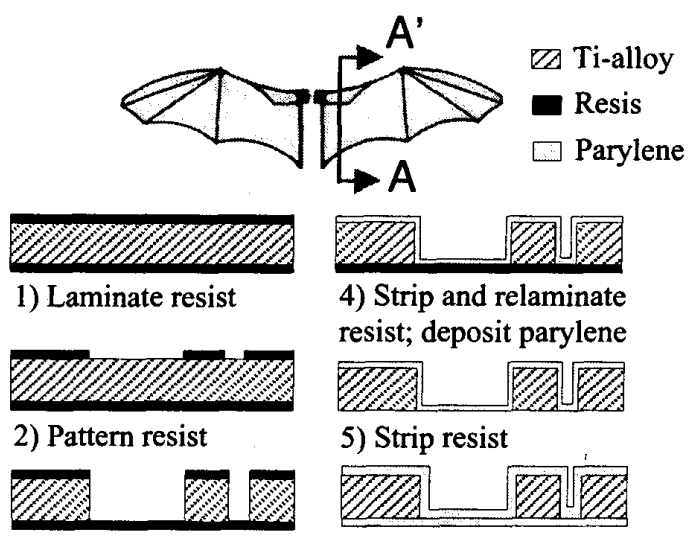

3) Etch Ti-alloy

6) Deposit backside parylene

Figure 6: Fabrication process of titaniumalloy MEMS wings

First, a $250-\mu \mathrm{m}$-thick titanium-alloy substrate is cleaned in trichloro ethylene (TCE) for 20 minutes. Later, it is dipped in a diluted HF solution to roughen the surface. Dry film resist is then laminated on both sides. The resist is patterned and hardbaked at $120^{\circ} \mathrm{C}$ for 20 minutes. The substrate is then etched in a solution of $\mathrm{HF}: \mathrm{HNO}_{3}: \mathrm{H}_{2} \mathrm{O}=5: 2: 100$ in volume. We find that if the concentration is stronger, the resist will peel off before the etching is finished. At this concentration, the etching rate is about $2.5 \mu \mathrm{m} / \mathrm{min}$. Since this is an isotropic etching, the undercut rate is about the same. Therefore, undercut must be taken into a consideration during the mask design.

After the etching process is finished and the wingframe is formed, the resist is stripped from both sides in a diluted $\mathrm{KOH}$ solution. Dry film resist is relaminated on the backside as a platform for parylene $\mathrm{C}$ polymers to deposit on. Parylence $\mathrm{C}$ is then deposited. Afterwards, dry film resist is stripped. Finally, in order to strengthen wing membrane, the second parylene $C$ deposition is performed.

We find that having the right material as a mask during etching is important. A crucial fabrication hurdle is releasing a large area of wing without damaging the membrane itself. The photoresist chosen must have an ability to withstand $\mathrm{HF}$ and $\mathrm{HNO}_{3}$ acids and can be stripped off without destroying the titanium-alloy metal and the parylene membrane. We selected negative dry film resist and found that its adhesion to titanium-alloy substrate was good. It could be stripped off easily in a diluted $\mathrm{KOH}$ solution if the film was exposed under the UV light beforehand. Both titanium-alloy wingframe and parylene membrane were also not attacked at all. 


\section{TESTING AND RESULTS}

\section{Mechanical Testing}

The wing stiffness test setup is shown in Figure 7. The wing is clamped at its root. A blade, connected to a loadcell and an XYZ stage, is used to probe at various section of the wings. A plot of spring vs. normalized distance from wing's root constant of a butterfly wing is shown in Figure 8. We see that MEMS wings can be tapered to vary thickness according to natural wing stiffness distribution. Tapered wings' stiffness, when normalized with weight, is also comparable to that of the natural wing.

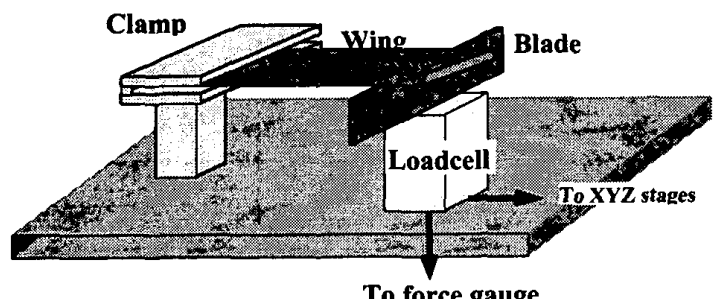

Figure 7: Spring constant test setup schematic

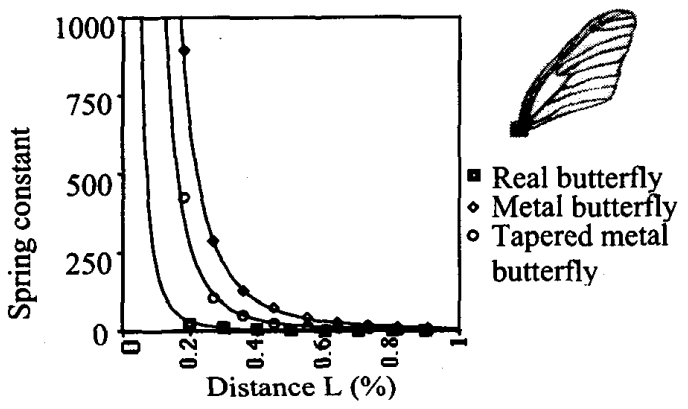

Figure 8: Spring constant of butterfly wings

\section{Transmission Design}

We built a lightweight, low-friction transmission mechanism to convert the rotary motion of the driving motor into the flapping motion of the wings based on simplicity, minimal weight, and flapping symmetry. This design restrict the flapping motion in a plane perpendicular to the motor shaft and is shown in Figure 9. A small DC motor with gearbox ratio of $22: 1$ was used to drive the transmission. MEMS wings were mounted on the transmission system and several flapping tests were performed. The wings could withstand more than $30 \mathrm{~Hz}$ of flapping without breaking. There were also no tears on the membrane.

\section{Wind Tunnel Test Results}

The MAV aerodynamic study was conducted at UCLA in a high quality low-speed wind tunnel with velocity uniformity of $0.5 \%$ and speeds from $1 \mathrm{~m} / \mathrm{s}$ to $10 \mathrm{~m} / \mathrm{s}$.

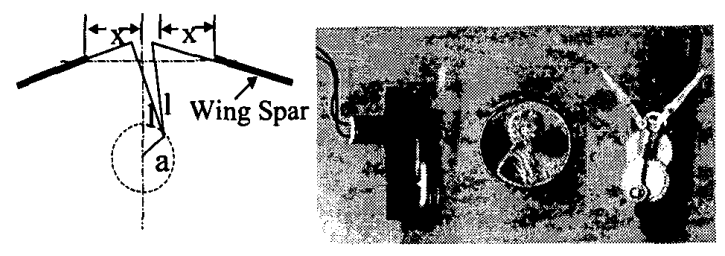

Figure 9: Transmission system

The wind tunnel has a $30 \times 30 \times 60 \mathrm{~cm}$ test section with a 4:1 contraction. Force measurements were taken using low capacity 2D force loadcells. The aerodynamic performance of natural insect wings, carbon fiber wings, and MEMS wings has been studied. As shown in Figure 10, wind tunnel test results show that spanwise stiffness is an important factor in lift production in flapping flight. For the same size of wings, cicada wings with rigid leading edge produce larger lift coefficients compared to our previous design of metal bat wings whose leading edge is flexible.

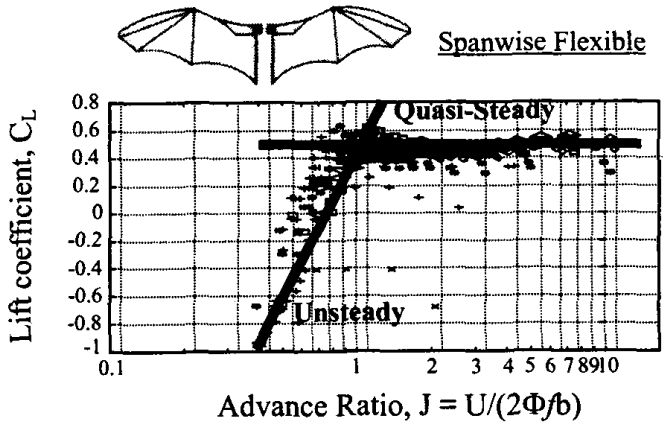

a) Titanium-alloy bat wings

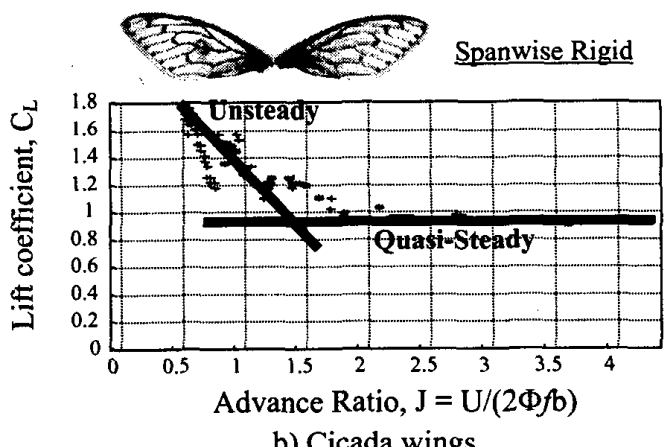

b) Cicada wings

Figure 10: Spanwise stiffness effect

The lift and thrust coefficients can be expressed as follows:

$$
\text { and } \begin{aligned}
C_{L} & =\frac{2 L}{\rho A U^{2}} \\
& C_{T}=\frac{2 T}{\rho A U^{2}}
\end{aligned}
$$


where $\mathrm{L}, \mathrm{T}, \mathrm{U}, \mathrm{A}, \rho$ are lift, thrust, flight speed, wing planform area, and air density, respectively. The advance ratio $\mathbf{J}$ is the ratio of the flight speed to the speed of the wingtip and is given by:

$$
J=\frac{U}{2 \Phi f b}
$$

where $\Phi, f, b$ are stroke angle, flapping frequency, and wing semi-span, respectively. Typically, unsteady-state flight has an advance ratio of less than 1 . For example, natural fliers such as bumblebee, black fly, and fruit fly have an advance ratio in free flight of $0.66,0.50$, and 0.33 , respectively [10].

Wind tunnel test results shows that nature-mimic wings with complicated structure performed poorly compared to wings with simple designs listed in Table 2. Thus, Lift and thrust coefficients for these simple-designed wings are shown in Figure 11. Figure 12 shows the input power required to flap these wings. Our current MEMS wing design D (CIT7 $\times 3$ S20) with rigid leadingedge shows the best result in terms of lift, thrust, and power required. It only needs 1 watt to flap at $30 \mathrm{~Hz}$.

Table 2: Properties of Various Wing Designs

\begin{tabular}{|l|c|c|c|c|}
\hline Wing types & A & B & C & D \\
\hline Weight (each), mg & 220 & 220 & 170 & 170 \\
\hline Frame material & $\mathrm{C}$ & $\mathrm{C}$ & $\mathrm{Ti}$ & $\mathrm{Ti}$ \\
\hline Membrane material & myl & pap & par & par \\
\hline Angle of diag. spa, deg & 45 & $\mathrm{n} / \mathrm{a}$ & 10 & 20 \\
\hline Planform LxW, cm & $7 \times 5$ & $7 \times 3$ & $7 \times 3$ & $7 \times 3$ \\
\hline
\end{tabular}

$\mathrm{C}=$ carbon fiber; $\mathrm{Ti}=\mathrm{T}$ itanium-alloy;

myl $=$ mylar; pap $=$ paper $;$ par $=$ parylene $C ; L=$ spanwise; $\mathrm{W}=$ chordwise
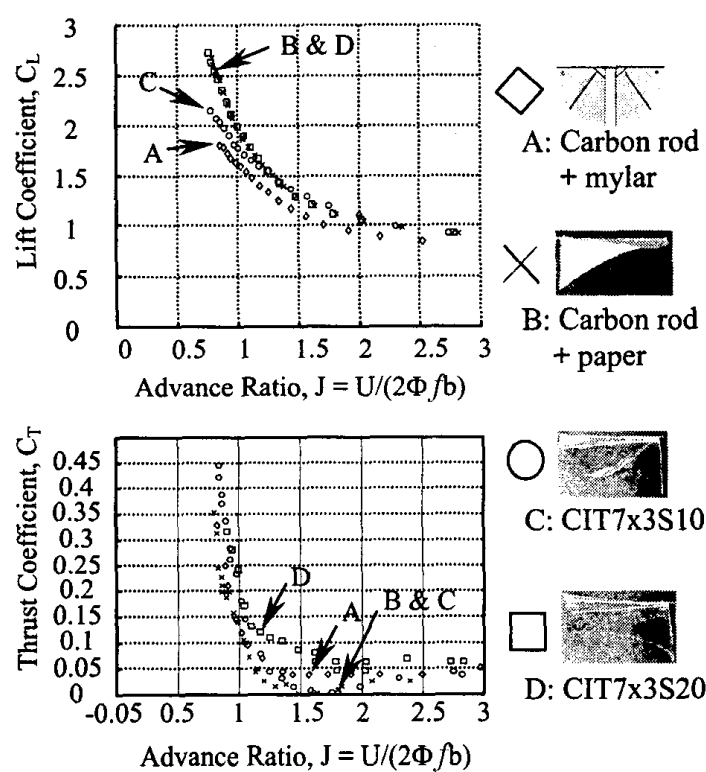

C: CIT7 73510

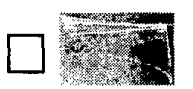

D: CIT7 3 S20

Figure 11: Lift and thrust coefficients of various types of wings

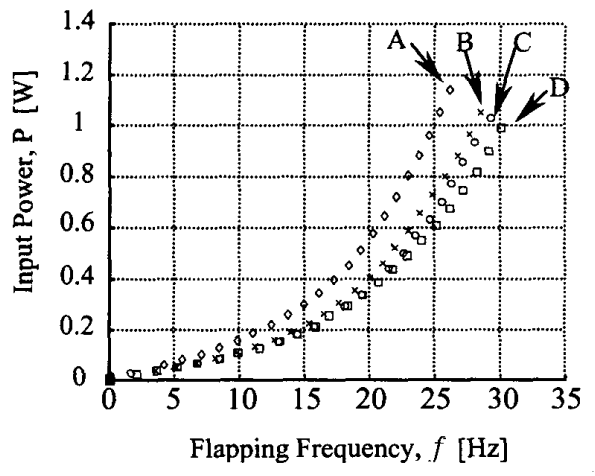

Figure 12: Input power

\section{PROTOTYPE VEHICLES}

\section{Super Capacitor-Powered Ornithopter}

We built a super capacitor-powered electric motor freeflight ornithopter, shown in Figure 13, which weighs only 6.5 grams. The system is composed of an electric motor, a transmission system, two 1-farad super capacitors, MEMS wings, a carbon-fiber-rod fuselage, and tail stabilizers. On the bench test, the flapping duration was less than a minute before having to recharge the capacitors. This is much shorter compared to the NiCd battery's discharge time.

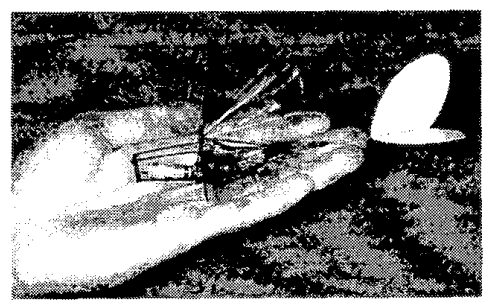

Figure 13: Super capacitor-powered ornithopter

\section{Battery-Powered Ornithopter}

Since our goal is to use a battery to provide a longer power source, we built a battery-powered ornithopter MAV as shown in Figure 14. We redesigned the body and replace super capacitors with a battery and a dc-to$\mathrm{dc}$ converter. The mass summary is shown in table 3 .

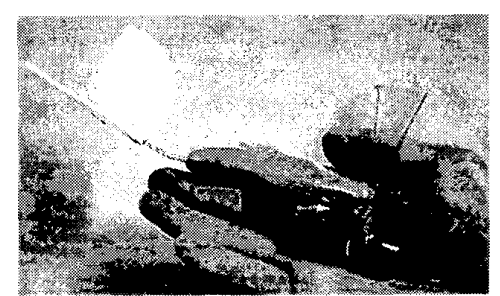

Figure 14: Battery-powered ornithopter MAV 
Table 3: Mass Summary for Battery-Powered Ornithopter

\begin{tabular}{|l|c|}
\hline Components & $\begin{array}{c}\text { Weight } \\
(\mathrm{g})\end{array}$ \\
\hline MEMS Wings & 0.4 \\
\hline Motor \& Transmission system & 3.1 \\
\hline Battery & 3.0 \\
\hline DC-to-DC Converter & 1.9 \\
\hline Fuselage, tail, switch, wires, etc. & 2.2 \\
\hline Total weight & 10.6 \\
\hline
\end{tabular}

The lightest rechargeable battery available we found is Sanyo NiCd N-50. It weighs about 3.5 grams. We trimmed the casing as thin as possible to reduce the weight to 3 grams. Since the NiCd battery produces only 1 volt nominally and the drive motor requires 4 to 6 volts, a DC-to-DC converter is custom-built to step up the voltage to the necessary 4 to 6 volt level to operate the electric motor. It weighs only 1.9 grams. The voltage output is adjustable and can be set before each flight test. The advantage of the converter and a $\mathrm{NiCd}$ cell power system is that it is light weight and takes advantage of the good specific power and specific energy of the $50 \mathrm{~mA}-\mathrm{hr}$ NiCd cell. We cannot use a higher quantity of smaller batteries to deliver the same power and performance due to the weight constraint.

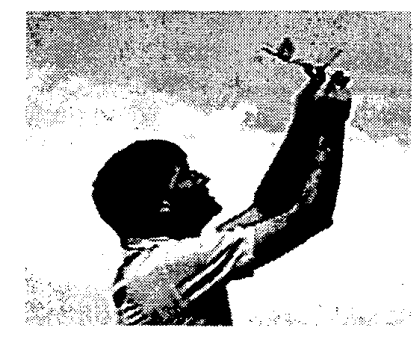

Figure 15: Recent flight test

\section{Flight Test}

A recent flight test is shown in Figure 15 . We have tested metal wings and carbon fiber wings with both ornithopters. Flight duration of 5 to 18 seconds were achieved. So far, the best flight duration for super capacitor-powered ornithopter was 9 seconds and 18 seconds for battery-powered ornithopter. The duration is mainly limited by the power system and vehicle's weight. In both cases, the metal wings did not break.

We find that there are several challenges in order to achieve a successful sustained flight. First, the wind condition must be perfect. Often during flight test, the wind speed and direction shifted constantly. Second, the trim of tail stabilizer must be crucial. Finally, each launch motion must be the same. We also believe that our current wings and ornithopter are not optimized, thus we hope future flight duration can be improved.

\section{CONCLUSION}

A novel titanium-alloy wingframe technology has been developed for MEMS wings. Several MEMS wings were fabricated with parameters, such as chord and spar widths, membrane thickness, number of spars, and sweep angle, varied. We believe that only MEMS technology can easily and systematically accommodate these many variable changes with a fast turn-around time. Wind tunnel tests were performed in the high quality wind tunnel at UCLA. Wings have been tested under cyclic conditions to assess long-term reliability. Super capacitor-powered and battery-powered ornithopters were built. The best free flight duration of 9 and 18 seconds were achieved by super capacitorpowered and battery-powered ornithopters, respectively.

\section{ACKNOWLEDGEMENTS}

This work is supported under DARPA/TTO MAV program DABT63-98-C-0005.

\section{REFERENCES}

[1] M. Okamoto, K. Yasuda, A. Azuma, “ Aerodynamic Characteristics of the Wings and Body of a Dragonfly", Journal of Experimental Biology, vol 199, pp. 281-294 (1996)

[2] M. Sato, A. Azuma, " The Flight Performance of a Damselfly Ceriagrion Melanuram Selys", Journal of Experimental Biology, vol 200, pp. 1765-1779 (1997)

[3] M. F. M. Osborne, “ Aerodynamic of Flapping Flight with Application to Insects", Journal of Experimental Biology, vol 28, pp. 221-245 (1951)

[4] C.H. Greenewalt, "Dimensional Relationships for Flying Animals", Smithsonian miscellaneous collections, vol 144, no. 2, pp. 1-46 (1962)

[5] C.J. Pennycuick, "The mechanics of bird migration”, IBIS, vol. 3, pp. 525-556 (1969).

[6] A. Azuma, "The Biokinetics of Flying and Swimming”, Springer-Verlag, Tokyo (1992)

[7] G.R. Spedding, "Advances in Comparative and Environmental Physiology", vol 11, pp. 51-111 (1992)

[8] American Society for Metals, "Metal Handbook", $9^{\text {th }}$ edition, vol 3, pp. 388-391 (1980)

[9] Product Specifications, “ Parylene Conformal Coatings Specifications and Properties", Specialty Coating Systems, Inc., Indianapolis (1994)

[10] S. Vogel, "Life in Moving Fluids", $2^{\text {nd }}$ edition., Princeton University Press, Princeton (1994) 